\title{
Aquisição da entoação em espanhol/L2: interação entre efeitos da instrução explícita e variáveis individuais
} \author{
explicit instruction and individuals variables \\ Elena Ortiz Preuss \\ Universidade Federal de Goiás - Goiânia, GO, Brasil \\ Rhanya Rafaella Rodrigues \\ Instituto Federal Goiano - Rio Verde, GO, Brasil \\ Wilson José de Oliveira Júnior \\ Universidade Federal de Goiás - Goiânia, GO, Brasil
}

Acquisition of intonation in Spanish/L2: interaction between the effects on

\begin{abstract}
Resumo: Este artigo expõe os efeitos da instrução explícita sobre enunciados interrogativos totais e asseverativos em espanhol como L2 e suas possíveis interações com variáveis individuais, como a capacidade de atenção, frequência de uso, experiência linguística e docente. Trata-se de um estudo quantitativo que abrange testes linguísticos de percepção e produção de enunciados, teste de rede de atenção e questionário de histórico e proficiência linguística. O estudo contou com uma amostra de conveniência abrangendo um grupo experimental que recebeu instrução explícita sobre o conteúdo-alvo num período intermediário entre o pré- e o pós-teste linguístico, e um grupo controle, que não recebeu a instrução. Os resultados mostraram que não houve diferença significativa, relacionada ao efeito da instrução. Nossa hipótese é de que as variáveis individuais possam ter minimizado o papel da instrução sobre entoação no desempenho desses participantes.
\end{abstract}

Palavras-chave: Instrução explícita; Diferenças individuais; Entoação

\begin{abstract}
This article exposes the effects of the explicit instruction on total interrogative and affirmative statements in Spanish as L2. It also displays their possible interactions with individual variables, such as the attention capacity, the frequency of use and also the linguistic and teaching experience. It is a quantitative study which covers some linguistic tests of perception and the production of sentences, an attention network test and a questionnaire of historical and linguistic proficiency. The study had a convenience sample covering both an experimental group that received some explicit instructions about a target content in an intermediate period between the pre- and the post-test, and a control group that did not receive the instruction. The results have shown that there was no significant difference related to the instructional effect. Our hypothesis is that individual variables may have minimized the instruction role about intonation in the performance of these participants.
\end{abstract}

Keywords: Explicit instruction; Individual differences; Intonation

\section{Introdução}

Muitas pesquisas sobre o processo de aquisição de segunda língua (doravante ASL) buscam observar os efeitos de diferentes tipos de instrução, comparando o desempenho de aprendizes, divididos em grupos experimentais e de controle, em testes linguísticos aplicados antes e depois do período instrucional. As condições pedagógicas do processo de ASL podem ser bastante diversas, como, por exemplo, aquisição em ambiente formal (instituições de ensino), informal (em casa ou com amigos) ou misto (simultaneamente numa instituição e fora dela); o tipo de input pode variar em quantidade (frequência de exposição) e qualidade; os tipos e modos de abordagem pedagógica podem prever instruções explícitas e/ou implícitas, distintas atividades de prática e de feedback, além de possibilidades diversas de interação. (SANZ, 2005). 
Em geral, tais estudos apontam evidências de efeitos significativos tanto em intervenções pedagógicas implícitas como explícitas (ELLIS, R., 2005; FINGER, PREUSS, 2009; SANZ, LIN, LADO, BOWDEN, STAFFORD, 2014; SANZ, 2005). Mas um aspecto interessante também identificado em algumas dessas pesquisas é que a magnitude dos efeitos parece depender de variáveis individuais. Em outras palavras, nem sempre as diferenças de desempenho dos participantes se devem, exclusivamente, aos efeitos do tipo de instrução testado. Bowden, Sanz e Stafford (2005) apresentam uma lista de diferenças individuais (doravante DIs) que podem afetar a aquisição de L2, na qual constam, por exemplo: idade, sexo, aptidão, motivação, atitude, personalidade, estilo cognitivo e de aprendizagem, capacidade de memória e de atenção, conhecimento prévio, entre outros.

Por outro lado, cabe salientar, sobre o ensino da entoação, que há ainda demanda por mais pesquisas que apontem quais abordagens (implícitas ou explícitas) seriam mais eficazes. Considera-se que o desenvolvimento da competência pragmática depende também do conhecimento de padrões melódicos adequados para o alcance de diferentes objetivos comunicativos. Nesse aspecto, a transferência de padrões da primeira língua (doravante L1) para a segunda língua (doravante L2), que é muito frequente (AKERBERG, 2011), pode gerar mal-entendidos e choques culturais, como fazer afirmação em vez de pergunta (enunciados enfocados neste artigo). Entretanto, em geral, no contexto de ASL a entoação tem sido tratada de forma mais implícita/ indireta (AKERBERG, 2011; CANTERO-SERENA, 2003; MORENO, 2002).

Em vista disso, propomos um estudo com vistas a verificar efeitos da instrução explícita na aquisição dos enunciados declarativos e interrogativos totais em espanhol/L2, bem como analisar se há correlação entre a instrução e variáveis individuais, como a frequência de uso, capacidade atencional e conhecimento prévio (experiência linguística e docente) dos participantes. $\mathrm{O}$ estudo, de natureza quali-quantitativa, caracteriza-se como semi-experimental e foi desenvolvido com aprendizes brasileiros de espanhol, que realizaram testes linguísticos e de atenção, além de preencher um questionário sobre seu histórico e proficiência linguística. Os dados foram tabulados e analisados estatisticamente.

Este artigo está dividido em cinco seções. Inicialmente, expomos o referencial teórico, em que discutimos a entoação em espanhol L2 e as variáveis externas e internas na ASL; em seguida, apresentamos os procedimentos de coleta e análise de dados; posteriormente, discutimos os resultados alcançados e expomos as considerações finais, seguidas das referências.

\section{Entoação em espanhol/L2}

A entoação é um elemento suprassegmental que abrange elementos linguísticos, paralinguísticos e extralinguísticos, além de aspectos como duração, intensidade e tom (RAE, 2011; SOSA, 1999). Parece haver um consenso no que se refere ao importante papel que é desempenhado pela entoação no âmbito da comunicação, visto que além de expressar aspectos linguísticos (diferentes tipos de enunciados), também permite a exteriorização de intenções comunicativas (por exemplo: pedido ou ordem), emoções (medo ou raiva) e outros aspectos inerentes ao falante (idade, gênero, variedade dialetal dentre outros) (CRYSTAL, 1970; PRIETO, 2003; SEMINO, 2014, dentre outros).

Akerberg (2011) e Dias (2015) argumentam que a entoação é muito significativa para a comunicação por contemplar elementos linguísticos e paralinguísticos, portanto, a realização de um contorno melódico que não se adeque aos padrões entoacionais da língua-alvo pode provocar problemas comunicativos entre os interlocutores. As autoras comentam que os enunciados declarativos e interrogativos totais são os que apresentam padrão entoacional mais diferente entre português brasileiro (PB) e espanhol, sendo que os interrogativos totais tendem a ser mais difíceis à aprendizes brasileiros de espanhol/L2.

Os enunciados declarativos são usados quando se quer explicar um pensamento ou dar alguma informação (RAE, 2011). A produção dos enunciados declarativos em espanhol é feita através da realização de um tom médio inicial, sucedido por diminuições progressivas no tom, após a primeira sílaba tônica, até um descenso total no tonema ${ }^{1}$ e pós-tonema. Salientamos que o movimento tonal entre o pré-tonema e o tonema é considerado semelhante ao de uma bola de tênis. Em outras palavras, o tom é inicialmente mais intenso seguido por um declínio gradual até o tonema (SOSA, 1999; SEMINO, 2014). A Figura $1^{2}$, adiante, ilustra o contorno melódico dos enunciados declarativos em espanhol ${ }^{3}$.

O padrão melódico dos enunciados declarativos em PB, observado na Figura 2, distingue-se do espanhol, unicamente, por apresentar movimento descendente contínuo, após a primeira sílaba tônica, conhecido como linha de declinação (LD), ao contrário do movimento de descenso gradual realizado no espanhol (PINTO, 2009).

\footnotetext{
Última sílaba tônica do enunciado, também denominada núcleo (SOSA, 1999).

2 Disponível em: <http://prosodia.upf.edu/atlasentonacion/enquestes/ espanol/madrid/index.html> Acesso em: 20 ago. 2016 (PRIETO; ROSEANO, 2009).

3 Destacamos que o padrão melódico pode variar conforme o dialeto ou a intencionalidade do falante (SOSA, 1999; RAE, 2011). No entanto, optamos por seguir o padrão mais recorrente entre as variedades, de modo a sistematizar a instrução explícita aplicada aos alunos.
} 


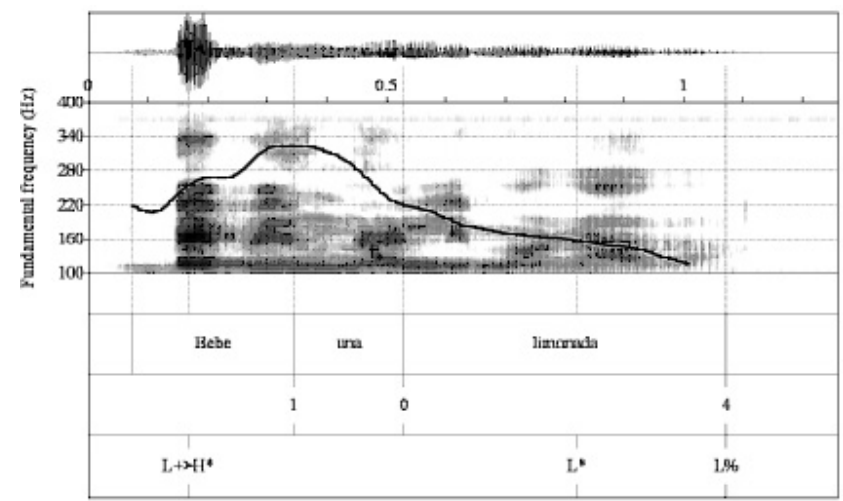

Figura 1. Padrão dos enunciados declarativos em Espanhol Fonte: Atlas interactivo de la entonación del español.

Kenuta pescava saxdmha.

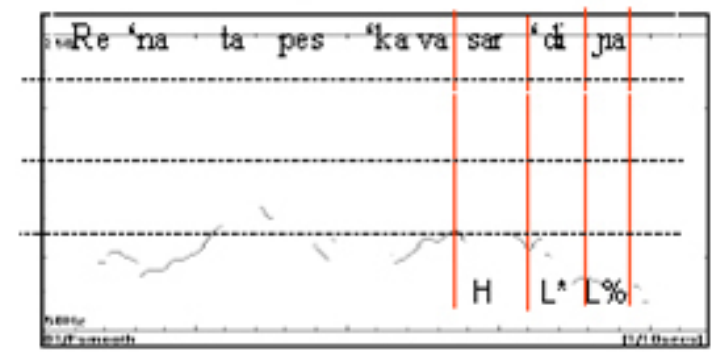

Figura 2. Padrão de enunciados declarativos no PB Fonte: Pinto, 2009, p. 42.

Os enunciados interrogativos podem ser denominados: i) parciais: que requerem informações do interlocutor e são introduzidos por uma partícula interrogativa e ii) totais: que apresentam respostas alternativas ou do tipo sim/não (SOSA, 1999; RAE, 2011). Salientamos que, neste estudo, enfocamos os interrogativos totais por apresentarem padrão entoacional distinto entre as línguas envolvidas, sobretudo, no que se refere à realização do tonema e pós-tonema, visto que o espanhol tende a apresentar contorno final ascendente $(\uparrow)$ e o PB contorno final circunflexo $\left(^{\wedge}\right)$ (AKEBERG, 2011; DIAS, 2015; PINTO, 2009).

Em espanhol, conforme ilustra a Figura $3^{4}$, as interrogativas totais são caracterizadas por apresentarem tom médio inicial, seguido por descenso entre a primeira sílaba tônica e o tonema, que é realizado com o tom ascendente (RAE, 2011).

Por sua vez, o PB, conforme Pinto (2009) é caracterizado por padrão melódico final circunflexo, ou seja, após o tonema há uma diminuição do tom, seguido por uma ascensão e, finalmente, um descenso tonal, como pode ser observado na Figura 4.

\footnotetext{
4 Disponível em: <http://prosodia.upf.edu/atlasentonacion/enquestes/ espanol/madrid/index.html> Acesso em: 20 ago. 2016 (PIETRO; ROSEANO, 2009).
}

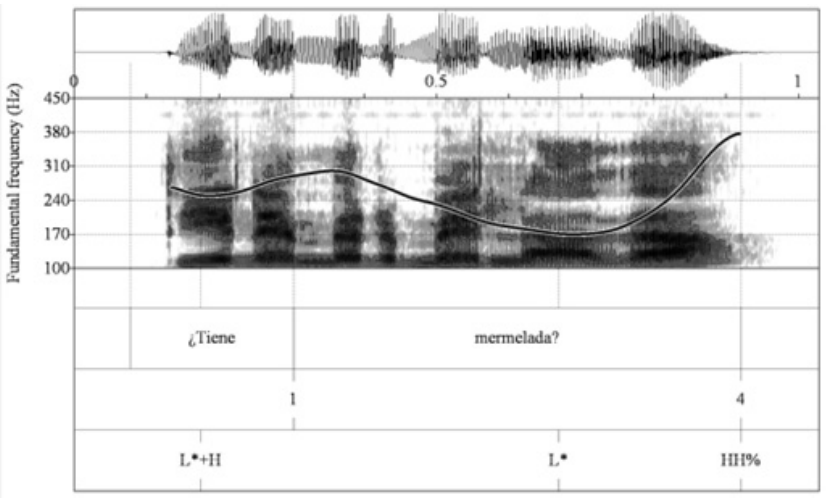

Figura 3. Interrogativos Totais em Espanhol Fonte: Atlas interactivo de la entonación del español.

\section{Renuta pescara sardinha?}

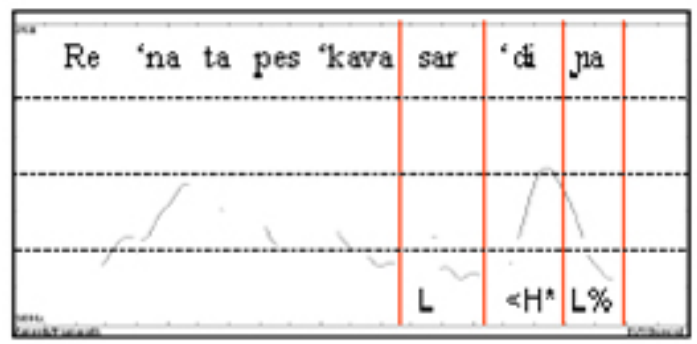

Figura 4. Interrogativos Totais em PB Fonte: Pinto, 2009, p. 66.

Argumenta-se também, comparando-se os padrões melódicos das duas línguas, que o espanhol tende a ter um tom inicial mais elevado, denominado por Sosa (1999) como predisposição inicializadora, em termos de pitch ${ }^{5}$, do que o PB (DIAS, 2015; PINTO, 2009). No Quadro 1, apresentamos uma síntese, com base em Semino (2014), das diferenças nos padrões entoacionais dos enunciados declarativos e interrogativos totais em espanhol e PB. Ressaltamos que focamos o tonema, por ser a parte do enunciado que concentra a maior diferença entre as línguas.

Quadro 1. Padrões entoacionais de enunciados em espanhol e PB

\begin{tabular}{|l|l|}
\hline Tipo de Oração & Tonema (última sílaba tônica) \\
\hline Declarativa em Espanhol & Diminuição do tom $(\downarrow)$ \\
\hline $\begin{array}{l}\text { Declarativa em Português } \\
\text { Brasileiro }\end{array}$ & Diminuição do tom (LD) $(\downarrow)$ \\
\hline Interrogativa Total em Espanhol & Aumento do tom ( $\uparrow)$ \\
\hline $\begin{array}{l}\text { Interrogativa Total em Português } \\
\text { Brasileiro }\end{array}$ & $\begin{array}{l}\text { Aumento e queda do tom na } \\
\text { pós-tônica }(\wedge)\end{array}$ \\
\hline
\end{tabular}

Fonte: adaptado de SEMINO, 2014.

\footnotetext{
5 Intensidade dos tons, medido através da F0.
} 
Embora a entoação seja um fenômeno importante e complexo, alguns autores argumentam que se trata de um aspecto que tende a ser ignorado ou tido como algo natural, espontâneo e inconsciente, sendo, consequentemente, abordado de forma implícita na sala de aula de L2 (AKERBERG, 2011; CANTERO-SERENA, 2003; MORENO, 2002).

Diferentes pesquisas sobre enunciados declarativos e interrogativos totais em espanhol têm sido realizadas nos últimos anos (AKERBERG, 2011; BODOLAY, 2011; COUTO; SÁ; FIGUEIREDO, 2013; DIAS, 2015; PINTO, 2009). Tais estudos focam aspectos relacionados à descrição dos enunciados e interferência entre as línguas. Notamos que a aquisição da entoação em espanhol/L2 também é um aspecto pouco explorado, em termos de pesquisa no campo da ASL. Desse modo, buscamos analisar efeitos de instrução explícita na aquisição da entoação em espanhol/L2.

\section{Variáveis externas e internas na ASL}

Nesta seção discutimos as interações entre variáveis (condições) externas, ou seja, as condições pedagógicas e as variáveis (diferenças) individuais dos aprendizes. As pesquisas na área de ASL tradicionalmente têm enfocado cada uma dessas variáveis isoladamente, na tentativa de encontrar uma teoria geral que explique totalmente o processo de aquisição, porém, há evidências mais atuais de que interações entre fatores externos e internos podem explicar melhor a grande variabilidade da proficiência, no caso de aquisição tardia da L2 (PREUSS; SANZ, 2016; SERAFINI e SANZ, no prelo).

Dentre os estudos com foco nas interações entre variáveis externas e internas algumas evidências se destacam, como, por exemplo, relações entre a idade dos aprendizes, a sua capacidade de memória de trabalho, a motivação e os efeitos de diferentes tipos de instrução (implícita, explícita, com ou sem feedback e prática) (COX e SANZ, 2015; LENET, et al., 2011).

Entre as condições pedagógicas, a instrução explícita tem sido bastante pesquisada e diversos autores destacam seus benefícios à ASL (ALVES, 2004; ELLIS, 2005; PREUSS, 2005). De acordo com Alves (2012), esse tipo de intervenção pedagógica, desenvolvida dentro de um contexto de ensino comunicativo, pode facilitar a formação de um saber de natureza explícita e contribuir para as práticas de automonitoramento pelo aprendiz. Para isso, é preciso que esteja atento e ciente da estrutura formal em questão. Sob essa perspectiva, a aprendizagem está relacionada com o grau de atenção dispensado na realização das atividades (SCHMIDT, 2001; IZUMI, 2013; WILLIAMS, 2013).
Schmidt (2001) argumenta que os mecanismos de atenção possuem capacidade limitada e são seletivos. Ou seja, é difícil manter nosso estado de atenção em alerta a tudo o que acontece ao nosso redor. No caso do desempenho linguístico, diferentes níveis de atenção são dispensados ao conteúdo e à forma de determinada mensagem, daí que ao focar o conteúdo, podemos produzir estruturas inadequadas, e ao focar a forma, provavelmente, haverá prejuízos ao conteúdo (fala mais lenta, esquecimentos ou omissões, por não saber como se expressar) (BYGATE, 2001; SCHMIDT, 2001; SKEHAN, 1996; VANPATTEN, 2005). Nesse sentido, a instrução explícita atuaria como uma forma de direcionamento atencional à determinada estrutura-alvo, presente no input ao qual o aprendiz é exposto.

\section{Método}

Nesta pesquisa visamos a observar efeitos da instrução explícita na aquisição de enunciados declarativos e interrogativos totais. Assim como, possíveis interações entre condições pedagógicas e variáveis individuais (capacidade atencional e experiência linguística e docente dos participantes). Trata-se de um estudo semiexperimental, de natureza quali-quantitativa. Participaram da pesquisa 9 aprendizes brasileiros de espanhol/L2, inseridos em um contexto de licenciatura em espanhol. Os participantes foram divididos em dois grupos, sendo um grupo experimental (GE), que contou com 5 alunos em nível intermediário, com média de idade de 27,5 anos e, um grupo controle (GC), que teve 4 participantes em nível intermediário, com média de idade de 23,2 anos. A opção por esses grupos deve-se à critérios de logística e conveniência: o grupo experimental é composto por acadêmicos do nível mais avançado do curso, o qual, pela primeira vez, oferece uma disciplina de língua com ênfase exclusiva na habilidade oral e com ensino explícito sobre entoação; por sua vez, o grupo controle é composto por egressos deste curso que não tiveram a oferta dessa mesma disciplina.

Após a assinatura do termo de consentimento livre e esclarecido $^{6}$, a pesquisa contou com as etapas que estão sintetizadas no Quadro 2, adiante.

Tanto pré- e pós-testes, quanto o teste ANT foram rodados no programa E-prime 2.0, que permitiu registrar a acurácia e o tempo de reação dos participantes. Os dados de produção foram segmentados com o auxílio do programa Audacity $2.1^{7} \mathrm{e}$, posteriormente, analisados no PRAAT, para que pudéssemos observar em detalhes

\footnotetext{
6 O protocolo deste estudo foi aprovado pelo CEP/UFG, sob o número CAAE 49155915.5.0000.5083

7 Trata-se de um software livre disponível para download em: <http:// www.audacityteam.org/download/>.
} 
Quadro 2. Procedimentos metodológicos

\begin{tabular}{|c|c|}
\hline Procedimento & Descrição \\
\hline $\begin{array}{l}\text { 1) Questionário de Proficiência e } \\
\text { Histórico Linguístico }\end{array}$ & $\begin{array}{l}\text { Composto por } 23 \text { perguntas relacionadas às informações gerais, ao histórico linguístico (experiência linguística } \\
\text { prévia) e de proficiência (uso e domínio das habilidades linguísticas) dos participantes. }\end{array}$ \\
\hline 2) Pré-Teste & $\begin{array}{l}\text { 1. Compreensão: Os participantes ouviram } 40 \text { enunciados (15 declarativos, } 15 \text { interrogativos totais e } \\
10 \text { negativos), tendo que indicar se eram afirmativos, interrogativos ou negativos*. } \\
\text { 2. Produção: Os testes de produção se dividiram em: produção controlada, semicontrolada e livre. } \\
\text { a. Produção controlada: leitura em voz alta de frases que apareciam na tela do computador ( } 10 \text { afirmativas, } \\
10 \text { interrogativas e } 5 \text { negativas). } \\
\text { b. Produção semicontrolada: produção de frases a partir das imagens que apareciam na tela do computador, } \\
\text { observando a pista do tipo de enunciado a ser produzido (A - afirmativo e ? interrogativo). Estava previsto a } \\
\text { produção de } 5 \text { enunciados afirmativos e } 5 \text { interrogativos. } \\
\text { c. Produção livre: produção de enunciados a partir das imagens que apareciam na tela do computador, sem } \\
\text { pistas (haviam } 6 \text { imagens). }\end{array}$ \\
\hline $\begin{array}{l}\text { 3) Tarefa de rede atencional } \\
\text { (Attentional Network task - ANT) }\end{array}$ & $\begin{array}{l}\text { Baseada em Costa, Hernández e Sebastián-Galles (2008), os participantes tinham que dizer para que lado } \\
\text { estava apontando a flecha central numa sequência, sob três condições: (1) condição congruente, quando a flecha } \\
\text { estava cercada de outras flechas que apontavam para o mesmo lado }(\rightarrow \rightarrow \rightarrow \rightarrow \rightarrow \text { ou } \leftarrow \leftarrow \leftarrow \leftarrow \leftarrow) \text {; ( } 2) \text { condição } \\
\text { incongruente, quando a flecha estava cercada de outras flechas, mas apontavam para direções diferentes } \\
(\rightarrow \rightarrow \leftarrow \rightarrow \rightarrow \text { ou } \leftarrow \leftarrow \rightarrow \leftarrow \leftarrow) \text {; e (3) condição neutra, quando flecha não estava cercada de outras flechas } \\
(+\rightarrow \text { ou }\end{array}$ \\
\hline 4) Instrução Explícita** & $\begin{array}{l}\text { Aconteceu durante duas semanas consecutivas, totalizando } 4 \text { aulas (duas vezes por semana, } 1 \text { h40min/cada). } \\
\text { Foram realizados os seguintes procedimentos pedagógicos: } \\
\text { 1. Introdução: A professora leu em voz alta enunciados, relativos ao tema viagem, alternando a entoação entre } \\
\text { afirmação e interrogação. Os alunos tinham que reagir às frases, a partir da entoação utilizada na leitura de cada } \\
\text { uma delas. } \\
\text { 2. Instrução explícita: A partir da leitura dos enunciados, a professora chamou a atenção dos alunos, para o fato } \\
\text { de que variações na entoação produzem reações distintas nos interlocutores. Posteriormente, chamou a atenção } \\
\text { de forma explícita para as diferenças nas curvas melódicas dos enunciados declarativos e interrogativos totais. } \\
\text { 3. Descriminação auditiva: Os alunos tinham que enumerar a sequência em que ouviam os enunciados } \\
\text { (declarativos e interrogativos totais). } \\
\text { 4. Produção controlada: Cada aluno tinha que ler as frases que havia recebido, considerando a entoação } \\
\text { sugerida através dos sinais de pontuação, e os demais colegas tinham que identificar a qual tipo de enunciado } \\
\text { se referia (interrogativo ou declarativo). A leitura dos enunciados pelos alunos foi gravada no programa } \\
\text { PRAAT**, a fim de que pudessem observar os contornos melódicos que produziram. } \\
\text { 5. Produção semicontrolada: Realizou-se a atividade "Busca a alguien que..., na qual os alunos tinham que } \\
\text { encontrar entre os colegas, quem já havia feito alguma das atividades propostas (ter viajado ao exterior, ter a } \\
\text { bagagem extraviada durante alguma viagem, etc). } \\
\text { 6. Produção livre: Os alunos leram previamente textos relacionados ao tema viagem (hospedar-se em albergues/ } \\
\text { hostels e fazer viagens como mochileiro), e realizaram um debate em sala de aula. }\end{array}$ \\
\hline 5) Pós-Teste & Seguia a mesmo padrão do pré-teste e foi aplicado após o período de instrução que o grupo experimental recebeu. \\
\hline
\end{tabular}

a curva melódica e realizássemos a análise, tendo como padrão o modelo métrico-autossegmental (SOSA, 1999). Esse padrão de análise categoriza os tons como altos ( $H$ - high) e baixos ( $L$-low), e considera os tons de núcleo $(*)$, que se referem à sílaba tônica, e de fronteira (\%), que são os que antecedem ou precedem o núcleo. A acuidade dos enunciados foi observada, utilizando-se o padrão $\mathrm{H}^{*} \mathrm{LL} \%$ ou $\mathrm{L} * \mathrm{~L} \%$, para os enunciados declarativos, e $\mathrm{H}^{*} \mathrm{H} \%$, para os interrogativos totais (SOSA, op.cit.; RAE, 2011), sendo atribuído o escore $\mathbf{0}$ para erro e 1 para acerto.

Todos os dados foram submetidos a análises estatísticas: descritiva, exploratória, inferencial e de correlação, através do programa SPSS 1.8. Na seção seguinte apresentamos a análise e discussão dos dados obtidos.

\section{Análise e discussão dos resultados}

Inicialmente realizamos testes de KolmogorovSmirnov e Shapiro-Wilk, os quais evidenciaram que a amostra não estava numa distribuição normal, por isso, foi feita a análise inferencial com o teste não-paramétrico de Wilcoxon, para comparar pré e pós-teste de cada grupo (intrasujeitos), e de Mann-Whitney, para comparar os resultados entre os grupos (intersujeitos). Nesta seção expomos as análises e discussões dos resultados obtidos nos testes de compreensão, de produção e as correlações com as variáveis individuais dos participantes.

\section{Compreensão de enunciados afirmativos e interrogativos totais}

O teste de compreensão continha 40 enunciados (15 afirmativos, 15 interrogativos totais e 10 negativos). Os participantes tinham que ouvir os enunciados e responder de que tipo eram, apertando as teclas correspondentes (A- se era afirmativo; $\mathbf{N}$ - se era negativo e ?- se era interrogativo). A tabela, a seguir, ilustra as médias e desvio padrão (DP), relativas à acurácia e ao tempo de reação (TR), medido em milissegundos. 
Tabela 1. Resultados dos testes de compreensão

\begin{tabular}{|c|c|c|c|c|c|}
\hline & & \multicolumn{2}{|c|}{ GE } & \multicolumn{2}{|c|}{ GC } \\
\hline & & $\begin{array}{c}\text { Pré-teste } \\
\text { Média (DP) }\end{array}$ & $\begin{array}{l}\text { Pós-teste } \\
\text { Média (DP) }\end{array}$ & $\begin{array}{c}\text { Pré-teste } \\
\text { Média (DP) }\end{array}$ & $\begin{array}{l}\text { Pós-teste } \\
\text { Média (DP) }\end{array}$ \\
\hline \multirow{2}{*}{ Afirmativas $(\mathrm{N}=15)$} & Acurácia & $14,20(0,83)$ & $14,60(0,89)$ & $13,75(0,50)$ & $15,00(0,00)$ \\
\hline & TR & $3138,85(274,22)$ & $2853,28(299,02)$ & $3284,18(325,90)$ & $2853,88(400,48)$ \\
\hline \multirow{2}{*}{ Interrogativas $(\mathrm{N}=15)$} & Acurácia & $13,80(1,78)$ & $14,20(1,78)$ & $14,25(0,50)$ & $15,00(0,00)$ \\
\hline & TR & $2864,74(254,95)$ & $2761,47(303,74)$ & $2800,76(355,62)$ & $2540,68(208,51)$ \\
\hline
\end{tabular}

Nota: $G E=$ grupo experimental; $C G=$ grupo controle; $M=$ média; $D P=$ desvio padrão; $T R=$ tempo de resposta.

De acordo com a Tabela 1, observe-se que, nos enunciados afirmativos, no pré-teste a maior média de acertos foi do GE ( $M=14,20)$, e no pós-teste foi do $\mathrm{GC}(\mathrm{M}=15,00)$. Ambos os grupos aumentaram os seus escores de acurácia no pós-teste, mas o maior aumento proporcional foi o do $\mathrm{GC}(\mathrm{M}=13,75$, no pré-teste, e $\mathrm{M}=15,00$, no pós-teste). Por sua vez, o GE apresentou leve aumento no DP $(0,83$, no pré-teste, e 0,89 , no pósteste), enquanto o GC diminuiu bastante o DP $(0,50$, no pré-teste, e 0,00 , no pós-teste). Com relação aos tempos de reação nos enunciados afirmativos, ambos os grupos demoraram mais para responder no pré-teste $(\mathrm{M}=3138,85$, do GE, e $M=3284,18$, do GC) do que no pós-teste ( $M=2853,28$, do GE, e $M=2853,88$, do GC), sendo que o GE foi o mais rápido dos grupos, nas duas etapas de teste, mas a diferença das médias dos tempos de reação entre os grupos diminuiu muito no pós-teste (de 145,33 no préteste, para 0,60 no pós-teste). Além disso, os escores de DP foram menores no GE $(274,22$, no pré-teste e 299,02 , no pós-teste $)$ do que no $\mathrm{GC}(325,90$, no pré-teste e 400,48 , no pós-teste), indicando que o comportamento dos participantes do GE era mais parecido do que os do GC.

Nos enunciados interrogativos, no pré-teste, as maiores médias de acertos foram do $\mathrm{GC}(\mathrm{M}=14,25$, no pré-teste, e $\mathrm{M}=15,00$, no pós-teste), sendo que os grupos aumentaram os seus escores entre o pré- e o pós-teste, mas os escores do GE sempre foram inferiores ao GC. Por outro lado, os escores de DP são maiores no GE (1,78 nas duas etapas de testes) enquanto que os do GC diminuiram $(0,50$, no pré-teste, e 0,00 , no pós-teste). Os tempos de reação nos enunciados interrogativos, de ambos os grupos, foram mais lentos no pré-teste ( $M=2864,74$, do $\mathrm{GE}$, e $\mathrm{M}=2800,76$, do $\mathrm{GC})$ do que no pós-teste ( $\mathrm{M}=2761,47$, do $\mathrm{GE}$, e $\mathrm{M}=2540,68$, do $\mathrm{GC})$, sendo que o $\mathrm{GC}$ foi o mais rápido dos grupos, nas duas etapas de teste, e a diferença das médias dos tempos de reação entre os grupos aumentou bastante (de 63,98 no pré-teste, para 220,79 no pós-teste). Além disso, os escores de DP diminuíram no GC $(355,62$, no pré-teste e 208,51 , no pós-teste) e aumentaram no $\operatorname{GE}(254,95$, no pré-teste e 303,4 , no pós-teste).
Nas análises de comparações de médias, tanto interquanto intra-sujeitos, não foram observadas diferenças significativas estatisticamente, entretanto, esses resultados podem estar evidenciando que: 1) o comportamento dos participantes pode ter sido modificado à medida que $\mathrm{o}$ grau de familiaridade com as tarefas aumentava, ou seja, as respostas eram mais rápidas e mais corretas no pósteste; 2) não foram detectados efeitos significativos da instrução, porque, em geral, o desempenho do GC foi melhor (mais acurácia e menor tempo de reação) do que o GE no pós-teste; 3) o comportamento do GE, cujo DP apresentou maior variação (aumento) no pós-teste, pode ser indício de que efeitos da instrução foram diferenciados entre os participantes, ou seja, o GE demonstra uma variação no comportamento, mas que ainda não teria se convertido numa aprendizagem consolidada; e 4) os enunciados interrogativos totais parecem ser mais fáceis de serem compreendidos do que os afirmativos. Cabe ressaltar que os enunciados interrogativos são os que mais se distinguem entre o espanhol e o português, mas, no geral, todos os grupos foram mais rápidos ao responder esses enunciados. A hipótese que formulamos é de que os participantes podem perceber logo no início que se trata de um enunciado interrogativo total, porque a curva melódica apresenta um tom inicial superior que aumenta após a última sílaba tônica, diferentemente, dos declarativos, cujo tom vai diminuindo até o fim do enunciado (DIAS, 2015; PINTO, 2009; SOSA, 1999). Passamos agora às análises das tarefas de produção.

\section{Produção de enunciados afirmativos e interrogativos totais}

Os testes de produção previam três tipos de tarefas: i) produção controlada, em que os participantes deviam ler em voz alta as frases que apareciam na tela do computador (havia 10 frases afirmativas; 10 interrogativas totais e 5 negativas), ii) produção semicontrolada, em que os participantes deviam criar frases, considerando a imagem e a pista (A ou ?, sendo A para enunciados afirmativos e ? para interrogativos) que aparecia no canto 
Tabela 2. Resultados dos testes de produção

\begin{tabular}{|c|c|c|c|c|c|}
\hline \multirow{4}{*}{ Afirmativas } & & \multicolumn{2}{|c|}{ GE } & \multicolumn{2}{|c|}{ GC } \\
\hline & & $\begin{array}{c}\text { Pré-teste } \\
\text { Média (DP) }\end{array}$ & $\begin{array}{l}\text { Pós-teste } \\
\text { Média (DP) }\end{array}$ & $\begin{array}{c}\text { Pré-teste } \\
\text { Média (DP) }\end{array}$ & $\begin{array}{l}\text { Pós-teste } \\
\text { Média (DP) }\end{array}$ \\
\hline & Controladas $(\mathrm{N}=10)$ & $\mathbf{8 , 2 0}(1,30)$ & $\mathbf{8 , 2 0}(1,30)$ & $9,25(0,50)$ & $\mathbf{8 , 7 5}(1,50)$ \\
\hline & Semicontroladas $(\mathrm{N}=5)$ & $2,75(1,50)$ & $2,80(1,09)$ & $3,00(1,15)$ & $3,75(1,25)$ \\
\hline \multirow{2}{*}{ Interrogativas } & Controladas $(\mathrm{N}=10)$ & $7,60(1,81)$ & $7,80(1,64)$ & $7,50(3,10)$ & $8,25(2,06)$ \\
\hline & Semicontroladas $(\mathrm{N}=5)$ & $2,20(0,83)$ & $2,25(1,50)$ & $1,33(1,15)$ & $\mathbf{2 , 7 5}(1,25)$ \\
\hline Produções livres & $(\mathrm{N}=6)$ & $3,20(1,09)$ & $2,03(4,44)$ & $3,75(1,25)$ & $4,25(0,85)$ \\
\hline
\end{tabular}

$\mathrm{GE}=$ grupo experimental; $\mathrm{GC}=$ grupo controle; $\mathrm{DP}=$ desvio padrão .

superior esquerdo da tela, (haviam 10 imagens, sendo 5 com a pista $\mathrm{A}$ e 5 com a pista ?); e iii) produção livre, em que os participantes deviam elaborar frases a partir de imagens (6 no total) que apareciam na tela, sem nenhuma pista; nesse caso, cabia ao participante decidir o tipo de enunciado a ser produzido.

Na Tabela 2, acima, estão expostos os dados relativos à acurácia dos participantes, no pré- e pós-teste de produção, através das médias e desvio padrão.

A Tabela 2 evidencia que nas frases afirmativas os maiores escores de correção nas produções controladas e semicontroladas foram do GC nos dois testes, mas enquanto nas controladas o GE manteve a mesma média de acertos e desvio padrão no pós-teste $(\mathrm{M}=8,20$, $\mathrm{DP}=1,30$ ), o GC diminuiu a média de acertos e aumentou o desvio padrão $(\mathrm{M}=8,75, \mathrm{DP}=1,50)$. Por outro lado, nas semicontroladas, houve aumento do número de acertos nos dois grupos no pós-teste $(\mathrm{GE}=2,80$ e $\mathrm{GC}=3,75)$.

Nos enunciados interrogativos, a média de acertos dos grupos, nas produções controladas, foi maior no pósteste, sendo que o escore do GC foi o maior de todos $(\mathrm{M}=8,25)$. O DP dos grupos diminuiu no pós-teste, mas os escores do GC continuaram maiores, o que parece indicar maior variação interna de desempenho dos participantes desse grupo. Nas produções semicontroladas, as maiores médias de acertos em enunciados interrogativos foram no pós-teste, sendo a média do GC $(\mathrm{M}=2,75)$ maior que a do GE $(\mathrm{M}=2,25)$. Os grupos também aumentaram os escores de desvio padrão, mas a maior variação foi no GE $(\mathrm{M}=1,50)$.

Nas produções livres, em que somente analisamos as produções dos enunciados-alvo ${ }^{8}$, é possível verificar que o GC apresentou os maiores escores de produções adequadas nos dois testes $(M=3,75$ no pré-teste e $M=4,25$, no pós-teste), e que o GE diminuiu o número de acertos no

\footnotetext{
8 Embora não seja possível discutir aqui, é importante mencionar que constatamos que nas produções livres, ambos os grupos preferiram produzir mais enunciados afirmativos ( $\mathrm{M}=5,4$ no $\mathrm{GE}$ e $\mathrm{M}=6,75$ no $\mathrm{GC})$ do que interrogativos ( $M=1$ no $G E$ e $M=2,75$ no GC). Note-se que a estrutura melódica das afirmativas é mais parecida ao português do que as interrogativas totais.
}

pós-teste $(\mathrm{M}=2,03)$, ao mesmo tempo em que apresentou o maior DP $(M=4,44)$. Note-se que, pelas médias de acertos, a instrução parece não ter tido efeito, mas o aumento do DP pode ser um indício de efeitos diferenciados entre os participantes, cuja aprendizagem das distinções entre os enunciados-alvo ainda não está consolidada.

Em geral, nas tarefas de produção o GC também mostrou maior desempenho (mais acurácia e menor tempo de reação) do que o GE, mas este apresentou mais variação no DP, o que pode ser um indício de que a instrução atingiu de forma diferenciada os participantes. Entretanto, todas essas comparações foram analisadas estatisticamente, mas não mostraram significância. Em vista disso, a hipótese é de que os efeitos da instrução podem ter sido afetados ou se relacionarem com variáveis individuais tais como, experiência linguística e docente, capacidade de atenção e frequência de uso. Disso trataremos na próxima subseção.

\section{Variáveis individuais e desempenho linguístico}

Na Tabela 3, adiante, são expostos os resultados do teste de atenção (ANT) e alguns dados do questionário de histórico e proficiência linguística sobre frequência de uso, experiência linguística e docente.

Conforme a Tabela 3, observe-se que no teste de atenção (ANT) ambos os grupos apresentaram médias gerais de acurácia muito próximas $(\mathrm{M}=31,33$, no $\mathrm{GE}$ e $\mathrm{M}=31,41$, no GC), sendo que o DP do GE é um pouco maior $(0,62)$. Com relação à média geral de tempo de reação (TR), no teste ANT, também se observa um comportamento muito parecido entre os participantes, sendo que o $\mathrm{GC}$ foi um pouco mais rápido $(\mathrm{M}=573,58)$, mas com DP maior $(170,57)$. As análises mostraram que as diferenças não foram significativas estatisticamente, evidenciando, assim que, em termos de capacidade de atenção, os dois grupos eram similares. Além disso, não foi observada correlação entre o desempenho dos participantes nas tarefas de percepção e produção de enunciados e os resultados do ANT. 
Tabela 3. Dados das variáveis individuais

\begin{tabular}{|c|c|c|c|}
\hline \multirow{3}{*}{ Atenção (Teste ANT) } & & $\begin{array}{c}\text { GE } \\
\text { Média (DP) }\end{array}$ & $\begin{array}{c}\text { GC } \\
\text { Média (DP) }\end{array}$ \\
\hline & Acurácia & $31,33(0,62)$ & $31,41(0,50)$ \\
\hline & TR & $579,17(101,47)$ & $\mathbf{5 7 3 , 5 8}(170,57)$ \\
\hline \multirow{2}{*}{ Frequência de uso } & \% uso diário & $57,00(24,39)$ & $20,25(22,41)$ \\
\hline & $\mathrm{N}^{\circ}$ horas diárias & $3,67(3,78)$ & $1,33(1,15)$ \\
\hline \multirow{5}{*}{ Experiência linguística } & $\%$ de contato atual & $81,00(18,16)$ & $47,50(34,76)$ \\
\hline & Escala de exposição atual & $7,04(1,21)$ & $5,20(1,78)$ \\
\hline & Escala de domínio das habilidades & $4,75(0,89)$ & $4,63(1,09)$ \\
\hline & Viver em país hispano-falante & $0,40(0,54)$ & $0,75(0,50)$ \\
\hline & Meses de imersão & $67,20(54,72)$ & $60,25(36,56)$ \\
\hline Experiência docente & Docência & $0,60(0,54)$ & $1,00(0,00)$ \\
\hline
\end{tabular}

$\mathrm{GE}=$ grupo experimental; $\mathrm{GC}=$ grupo controle; $\mathrm{DP}=$ desvio padrão.

Com relação à frequência de uso, avaliada tanto pelo percentual de uso diário, quanto pelo indicativo de número de horas diárias em que a L2 é utilizada, observase que o GC apresenta escores menores de uso. Os testes de Correlação de Pearson, aplicados para verificar a associação entre o desempenho dos participantes nos testes linguísticos e a frequência de uso, mostrou relação entre o número de horas diárias e a acurácia nas interrogativas semicontroladas do GE $(r=-1,000$, $\mathrm{p}<0,00$ ), sendo, neste caso, uma correlação negativa em que quanto mais horas diárias, menor era o número de acertos. Houve associação entre o tempo de reação na compreensão das interrogativas em ambos os grupos (GE: $r=0,998, p<0,03$; GC: $r=-0,997, p<0,05)$; no GE a correlação foi positiva, ou seja, quanto mais horas diárias, maior era o tempo de reação, e no $\mathrm{GC}$, a correlação era negativa, ou seja, quanto menos horas diárias, menor era o tempo de reação.

Para avaliar a experiência linguística, levamos em consideração os seguintes itens: porcentagem (0 a 100\%) de contato atual; escala (de 0 a 10) de exposição atual; domínio geral, obtido através de uma média dos escores nas habilidades de leitura, escrita, audição e fala, em uma escala de 1 a 6; e imersão, por meio do indicativo do número de meses em situação de imersão, seja, em casa, na escola ou outro ambiente em que a L2 era falada, e por meio do indicativo de ter ou não vivido em algum país em que a L2 era falada. Note-se que os escores do GC são sempre inferiores aos do GE em quase todos os itens avaliados para a experiência linguística, a única exceção é no item viver em país hispano-falante. Os testes de Correlação de Pearson, aplicados para verificar a associação entre o desempenho dos participantes nas tarefas de percepção e produção de enunciados e a experiência linguística, apontaram que houve correlação significativa entre contato atual e desempenho, no GE, na produção de interrogativas semicontroladas $(r=-0,920$, $\mathrm{p}<0,02$ ), em que a correlação foi negativa, quanto maior o contato, menor era a acurácia; na compreensão de interrogativas $(r=0,914, p<0,03)$ e afirmativas $(r=0,930$, $\mathrm{p}<0,02$ ), em que as correlações foram positivas, pois quanto maior o contato, maior a acurácia da compreensão. Foram observadas associações entre exposição atual e tarefas envolvendo interrogativas, como no caso do GE, na produção de interrogativas semicontroladas $(r=-0,978, p<0,004)$, em que foi negativa, ou seja, quanto mais exposição menos acurácia; por outro lado na compreensão das interrogativas $(r=0,901, p<0,03)$ houve correlação positiva, isto é, quanto maior a exposição, maior é a acurácia. No GC, constatamos uma correlação negativa $(r=-0,958, p<0,04)$ entre a exposição e o tempo de resposta na compreensão das interrogativas, isto é, quanto mais exposição menor é a latência da nomeação.

Com relação à experiência docente, cuja média do GC foi superior ao GE, observamos uma correlação positiva $(r=0,932, p<0,02)$ entre a docência e o desempenho dos participantes na produção de afirmativas semicontroladas no GE, ou seja, quem tinha experiência docente obteve maior acurácia na produção desses enunciados. Considerando-se que não foram identificados efeitos significativos da instrução, esses resultados das variáveis individuais em relação às tarefas linguísticas parecem sinalizar que ter vivido em um país que fala a L2 ou ter experiência docente podem contribuir para o desenvolvimento da percepção das distinções entre enunciados interrogativos totais e enunciados afirmativos em português e espanhol. Além disso, é preciso ponderar que no GE também havia participantes com experiência docente o que dificulta ainda mais a identificação de efeitos significativos da instrução, demonstrando que talvez a logística da distribuição entre grupo controle e experimental, apesar de ter sido motivada por razões de conveniência, pode ter afetado os resultados. Do mesmo 
modo, como se trata de uma amostra com número reduzido de participantes, a detecção de diferenças estatisticamente significativas é dificultada.

Um aspecto interessante dos resultados é que frequência de uso e tempo de contato atual parece não ajudarem no melhor desempenho linguístico (nos referimos às associações entre mais uso e contato com menos acurácia e mais tempo para iniciar a resposta) dos participantes. Algo um pouco contraditório, considerando-se a literatura sobre ASL, em que se defende a importância da frequência e da prática na aquisição (ELLIS, 2005; PREUSS, 2005). Mas esse fato e a variação no DP do GE nos parecem indícios de que a instrução afetou diferentemente os participantes, mas ainda não foi suficiente para consolidar a aprendizagem. Em vista disso, ressaltamos a necessidade de mais pesquisas, com uma amostra maior, inclusive, prevendo-se coletas em outros ambientes de ensino com controle de variáveis individuais.

\section{Considerações finais}

Este estudo tinha por objetivo analisar os efeitos da instrução explícita sobre enunciados interrogativos totais e asseverativos em espanhol como L2 e suas possíveis interações com variáveis individuais (capacidade de atenção, frequência de uso, experiência linguística e docente). Em geral, os testes de comparação não apontaram diferenças significativas para os efeitos da instrução. Observamos também que não houve interação entre o desempenho linguístico dos participantes e a capacidade atencional. Por outro lado, houve algumas correlações envolvendo outras variáveis individuais, tais como frequência de uso, experiência linguística e docente, em que se destacam o número de horas diárias, o percentual de contato atual, a escala de exposição atual e a docência.

Aparentemente, variáveis individuais como docência e imersão através da vivência em países que falam a L2-alvo podem ter efeitos compatíveis com a instrução explícita em ambiente formal. Entretanto, devido à amostra reduzida, não é possível detectar diferenças significativas estatisticamente, de modo a tecer considerações mais fundamentadas. Parece-nos pertinente e necessário o desenvolvimento de mais pesquisas enfocando os efeitos de instrução explícita sobre a entoação em interação com as variáveis individuais.

\section{Referências}

AKERBERG, Marianne. Aquisição da Pronúncia: a entoação em afirmações e perguntas sim/não. Trabalhos em Linguística Aplicada, Campinas, v. 50, n. 1, p. 153-167, 2011.
ALVES, Ubiratã Kichhöfel. A explicitação dos aspectos fonético-fonológicos da L2: teoria e pesquisa na sala de aula. In: LAMPRECHT, Regina R. (Org.). Consciência dos sons da língua: subsídios teóricos e práticos para alfabetizadores, fonoaudiólogos e professores de língua inglesa. 2. ed. Porto Alegre: EDIPUCRS, 2012. p. 211-230.

ALVES, Ubiratã Kichhöfel. O papel da instrução explícita na aquisição fonológica do inglês como L2: evidências fornecidas pela Teoria da Otimidade. 2004. Dissertação (Mestrado em Letras) - Escola de Educação, Universidade Católica de Pelotas, Pelotas, 2004.

BODOLAY, Adriana. Análise prosódica de línguas em contato: questões totais no português e no espanhol falado na fronteira Brasil/Uruguai. In: Anais do Colóquio Brasileiro de Prosódia da Fala. Belo Horizonte: Periódicos UFMG, 2011. Vol. 1. p. 8-14. Disponível em: <http://www.periodicos.letras.ufmg. br/index.php/anais_coloquio/article/view/1199/1348>. Acesso em: 13 set. 2016.

BYGATE, Martin. Speaking. In: CARTER, Ronald. NUNAN, David. (Ed.). The Cambridge guide to teaching English to speakers of other languages. Cambridge: Cambridge University Press, 2001. p. 14-20.

CANTERO SERENA, Francisco José. Fonética y didáctica de la pronunciación. In: MENDOZA, A. (Coord.). Didáctica de la lengua y la literatura. Madrid: Prentice Hall, 2003. p. 545-572.

COSTA, Albert; HERNÁNDEZ, Mireia; SEBASTIÁNGALLÉS, Núria. Bilingualism aids conflict resolution: evidence from the ANT task. Cognition, Elsevier Science B.V., v. 106, n. 1, p. 59-86, 2008.

COUTO, Letícia Rebollo; SÁ, Priscila Ferreira de; FIGUEIREDO, Natalia dos Santos. Gêneros orais e entoação: os enunciados interrogativos no espanhol de Buenos Aires e Montevidéu. Interdisciplinar, Itabaiana-SE, n. VIII, v. 17, p. 69-98, 2013.

COX, Jessica G.; SANZ, Cristina. Deconstructing PI for the ages: Explicit instruction vs. practice in young and older adult bilinguals. IRAL - International Review of Applied Linguistics. v. 53, n. 2, p. 225-248, 2015.

CRYSTAL, David. Prosodic Systems and Language acquisition. In: LEON, Pierre R.; FRAURE, Georges; RIGAULT, Andre. (Ed.). Prosody Feature Analysis. Montreal: Didier, 1970. p. 77-90.

DIAS, Eva Christina Orzechowski. Declarativas e interrogativas totais no espanhol L1 e L2 falado em Bogotá: uma contribuição para estudos prosódicos. 2015. $273 \mathrm{f}$. Tese (Doutorado em Linguística) - Programa de Pós-Graduação em Linguística, Universidade Federal de Santa Catarina, Florianopólis. 2015.

ELLIS, Rod. La adquisición de segundas lenguas en un contexto de enseñanza análisis de las investigaciones existentes. Nova Zelandia: Research Division, 2005.

FINGER, Ingrid; PREUSS, Elena Ortiz. Atenção ao input e aprendizagem: o papel da instrução explícita na aquisição do Espanhol como L2. Letras de Hoje, v. 44, n. 3, p. 78-85, 2009.

FORTKAMP, Mailce Borges. Produção oral e aquisição de L2: cognição e ensino. In: MATZENAUER, Carmen Lúcia Barreto et al. (Org.). Estudos da Linguagem: VII Círculo de Estudos Linguísticos do Sul. Pelotas: Educat, 2008. p. 223-234. 
IZUMI, Shinichi. Noticing and L2 Development: Theoretical, Empirical, and Pedagogical Issues. In: BERGSLEITHNER, Joara M.; FROTA, Sylvia Nagem; YOSHIOKA, Jim K. (Ed.). Noticing and Second Language Acquisition: Studies in Honor of Richard Schmidt. Honolulu: National Foreing Language Resource Center, 2013. p. 25-38.

LENET, Alison E.; SANZ, Cristina; LADO, Beatriz; HOWARD Jr, James H.; HOWARD, Darlene V. Aging, pedagogical conditions and differential success in SLA: an empirical study. In: SANZ, Cristina; LEOW, Ronald (Ed.). Implicit and explicit language learning: conditions, processes and knowledge in SLA and bilingualism. Washington DC: Georgetown University Press, 2011. p. 73-84.

PREUSS, Elena Ortiz. A interface entre os conhecimentos implícito e explícito: um estudo baseado no se-operador aspectual como delimitador em espanhol. 2005. 165 f. Dissertação (Mestrado em Letras) - Universidade Católica de Pelotas, Pelotas, 2005.

PREUSS, Elena Ortiz; SANZ, Cristina. Aquisição de L2: Interação entre variáveis externas e internas. In: PREUSS, Elena Ortiz; COUTO, Elza Kioko Nakayama N. do; RAMOS, Rui Manuel (Org.). Multiplos olhares e Linguística e Linguística Aplicada. Campinas: Pontes, 2016. p. 121-134.

PINTO, Maristela da Silva. Transferências prosódicas do $\mathrm{PB} / \mathrm{LM}$ na aprendizagem do E/LE: enunciados assertivos e interrogativos totais. 2009. 378f. Tese (Doutorado em Letras Neolatinas) - Universidade Federal do Rio de Janeiro, Rio de Janeiro, 2009.

PRIETO, Pilar. Teorías lingüísticas de la entonación. In: PRIETO, Pilar. Teorías de la Entonación. Barcelona: Ariel, 2003. p. 14-33.

RAE. Fonética y fonología. Cuestiones generales. In: RAE. Nueva gramática de la lengua española. Barcelona: Espasa Libros, 2011. p. 19-22.

RAE. La entonación In: RAE. Nueva gramática de la lengua española. Barcelona: Espasa Libros, 2011. p. 435-488.

SANZ, Cristina. Contributions of study abroad research to our understanding of SLA processes and outcomes: The SALA Project, an appraisal. In: PÉREZ-VIDAL, Carmen (Ed.). Language acquisition in study abroad and formal instruction contexts. Philadelphia/Amsterdam: John Benjamins, 2014.p. 1-13.
SANZ, Cristina; SERAFINI, Ellen Johnson. Cognición y L2: El papel de las diferencias individuales en el aprendizaje de lenguas extranjeras con especial atención a la memoria operativa. No prelo.

SANZ, Cristina. Adult SLA: the interaction between external and internal factors. In: SANZ, Cristina (Ed.). Mind and Context in adult second language acquisition: methods, theory, and practice. Washington DC: Georgetown University Press, 2005. p. 3-20.

SCHMIDT, Robert. Attention. In: ROBINSON, Peter (Ed.). Cognition and second language instruction. Cambridge University Press, 2001. p. 3-32.

SEMINO, María Josefina Israel. La entonación en español y en portugués. In: BRISOLARA, Luciene Bassols; SEMINO, María Josefina Israel. ¿Cómo pronunciar el español? La enseñanza de la fonética y la fonología para brasileños: ejercicios prácticos. Campinas: Pontes Editores, 2014. p.103-126.

SKEHAN, Peter. A. Framework for the implementation of task-based instruction. Applied Linguistics, v. 17, n. 1, p. 38-62, 1996.

SOSA, Juan Manuel. La entonación del español. Su estructura fónica, variabilidad y dialectología. Madrid: Cátedra, 1999.

VANPATTEN, Bill. Processing Instruction. In: SANZ, Cristina. (Ed.). Mind and Context in adult second language acquisition: methods, theory, and practice. Washington DC: Georgetown University Press, 2005. p. 267-281.

WILLIAMS, John N. Attention, Awareness, and Noticing in Language Processing and Learning. In: BERGSLEITHNER, Joara M.; FROTA, Sylvia Nagem; YOSHIOKA, Jim K. (Ed.). Noticing and Second Language Acquisition: Studies in Honor of Richard Schmidt. Honolulu: National Foreing Language Resource Center, 2013. p. 39-57.

Recebido: 10 de janeiro de 2017 Aprovado: 23 de março de 2017

Contato:

Elena Ortiz Preuss <elena.ortizp@yahoo.com.br>

Rhanya Rafaella Rodrigues <rhanyarafaella@gmail.com $>$

Wilson José de Oliveira Júnior <wilsonufg.letras@gmail.com> 